\title{
sdRNA: siRNA with a DNA Seed for an Efficient and Target-gene Specific RNA Interference
}

\section{Kumiko Ui-Tei*}

Department of Biophysics and Biochemistry, Graduate School of Science, The University of Tokyo, 7-3-1 Hongo, Bunkyo-ku, Tokyo 113-0033, Japan

\begin{abstract}
RNA interference (RNAi), a process through which small interfering RNAs (siRNAs) induce sequence-specific post-transcriptional gene silencing, is commonly recognized as a powerful tool not only for functional genomics but also for therapeutic applications. To achieve accurate target gene function and successful therapeutic applications, it is necessary to select an efficient and target genespecific siRNA with minimal off-target effects. We found that the ability to induce off-target effects on unintended genes is strongly correlated to the thermodynamic stability of the duplex formed between the seed region (positions 2-8 from the 5' end of the siRNA guide strand) and target mRNA. Consistent with this property, we found that DNA-RNA chimeric siRNA (chiRNA) with deoxyribonucleotides in the 5' proximal eight nucleotides of the guide strand and the complementary nucleotides in the passenger strand exerted virtually no off-target effect due to low stability of the DNA-RNA duplex in seed-target base-pairing. However, the corresponding RNAi activities for primary target genes were also decreased to one-tenth on average by the DNA substitutions. Here, we report that siRNAs with seven deoxyribonucleotides exclusively in the seed region (sdRNA) may exhibit efficient target-specificity, but off-target effect-reduced RNAi activity.
\end{abstract}

Keywords: DNA modification; Off-target effect; Seed region; Small interfering RNA; siRNA; RNA interference; RNAi

\section{Introduction}

RNA interference (RNAi) is an evolutionarily conserved pathway induced by small interfering RNAs (siRNAs), which are $19 \mathrm{bp}$ in length, double-stranded RNAs (dsRNAs) with two-nucleotide 3' overhangs [1]. siRNAs incorporated into cells are transferred to an RNAi effector complex called an RNA-induced silencing complex (RISC) [2,3]. RISCs are assembled on one of the two strands of the siRNA duplex and are activated upon removal of the passenger strand [4-6]. Others reported that asymmetric features at both siRNA terminals are common to functional siRNAs [6-8]. An RNA strand with an unstable 5' terminus is easily retained in a RISC. An activated RISC is a ribonucleoprotein complex consisting minimally of a core protein, Argonaute (Ago) and an siRNA guide strand [9-11]. The siRNA guide strand retained in an RISC recognizes mRNAs with perfectly complementary sequences and the Ago2 protein cleaves them.

The RNAi effect of a siRNA has been assumed to be extremely specific. However, accumulated evidence has revealed that siRNAs may downregulate many unintended genes with partial complementarities mainly in the seed region (nucleotides 2-8 from the 5' end of the siRNA guide strand) [12-16]. This phenomenon is referred to as an off-target effect. The recognition mechanism of an off-target effect is known to be similar to that of microRNA-mediated gene silencing [13-15,1721]; seed nucleotides are present on the Ago surface in a quasi-helical form to serve as the entry or nucleation site for small RNAs in the RISC $[12,22,23]$. To achieve accurate target gene function and successful therapeutic applications, it may be critical to select a target genespecific siRNAs with minimal off-target effects.

We found that ability to induce an off-target effect is strongly correlated to the thermodynamic stability of the duplex formed between the seed region of siRNA guide strand and target mRNA (seed-target duplex) (Figure 1a) [12]. Furthermore, we have developed a DNARNA chimeric siRNA (chiRNA) with deoxyribonucleotides in the 5 proximal eight nucleotides of the guide strand and the complementary nucleotides. The chiRNA showed virtually no off-target effects, probably because the stability of the seed-target duplex was reduced by the DNA substitutions in the siRNA seed region (Figure 1b) [16]. For target-specific RNAi with reduced off-target effects, we propose an improved chiRNA, referred to as an sdRNA, in which seven nucleotides in the seed region is exclusively replaced with DNA without including a nucleotide at the 5 terminus (Figure 1c).

\section{Materials and Methods} siRNA

RNAi efficiency in mammalian cells varies considerably depending on the siRNA sequence. We have shown that highly functional siRNAs for mammalian RNAi have A or $\mathrm{U}$ residues at nucleotide position 1 measured from the $5^{\prime}$ end of the guide strand, four to seven A/Us at nucleotide positions 1-7 ( $\mathrm{AU} \geq 57 \%$ ) and a $\mathrm{G} / \mathrm{C}$ at position 19 (Figure 1) [6]. In addition, a GC stretch of no more than nine nucleotides is contained in siRNA sequences. All of the double-stranded small RNAs used in this study shown in S1 simultaneously satisfied these conditions.

\section{Cell culture and silencing assay}

Human HeLa cells were cultured and subjected to gene silencing as described previously [6,12]. Briefly, a $1 \mathrm{ml}$ suspension of human HeLa cells $\left(1 \times 10^{5}\right.$ cells $\left./ \mathrm{ml}\right)$ was inoculated in a $1.5 \mathrm{~cm}$ well $24 \mathrm{~h}$ prior to transfection. The cells were transfected with pGL3 or pGL2 control ( $1 \mu \mathrm{g}$; Promega), both of which encode firefly luciferase (luc), and the Renilla luc-encoding plasmid psiCHECK-completely matched (CM) or seed matched (SM) $(0.1 \mu$; Promega) with siRNA, chiRNA, sdRNA, or sd/chiRNA. Lipofectamine 2000 regent (Invitrogen) was used for transfection. Each of siRNA, chiRNA, sdRNA, or sd/chiRNA $(0.5,5$, $50,500,5,000$, and $50,000 \mathrm{pM}$ ) was diluted in $50 \mu \mathrm{l}$ of Opti-MEMI without fetal bovine serum (FBS) and mixed with $50 \mu \mathrm{l}$ Opti-MEMI containing $1 \mu \mathrm{l}$ of Lipofectamine 2000 for $30 \mathrm{~min}$ in room temperature. After the culture medium was changed with FBS-free medium, 100 $\mu l$ of siRNA, chiRNA, sdRNA, or sd/chiRNA-Lipofectamine 2000 complex were added to each well. After $4 \mathrm{~h}$ incubation, the medium was replaced with fresh medium with FBS. The cells were harvested

*Corresponding author: Kumiko Ui-Tei, Department of Biophysics and Biochemistry, Graduate School of Science, The University of Tokyo, 7-3-1 Hongo, Bunkyo-ku, Tokyo 113-0033, Japan, Tel: +81 35841 3043; Fax: +81 35841 3043; E-mail: ktei@bi.s.u-tokyo.ac.jp

Received October 27, 2012; Accepted November 20, 2012; Published November 26, 2012

Citation: Ui-Tei K (2012) sdRNA: siRNA with a DNA Seed for an Efficient and Target-gene Specific RNA Interference. Gene Technol 1:102. doi:10.4172/2329-6682.1000102

Copyright: (c) 2012 Ui-Tei K. This is an open-access article distributed under the terms of the Creative Commons Attribution License, which permits unrestricted use, distribution, and reproduction in any medium, provided the original author and source are credited. 
Citation: Ui-Tei K (2012) sdRNA: siRNA with a DNA Seed for an Efficient and Target-gene Specific RNA Interference. Gene Technol 1:102. doi:10.4172/2329-6682.1000102

(a)

\section{SiRNA-mediated silencing}
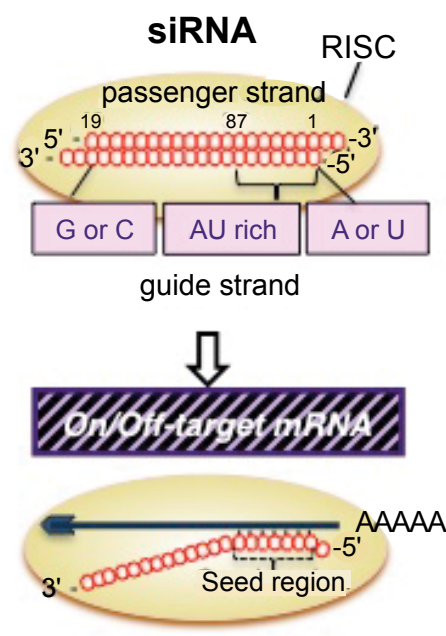

Strong base-pairing

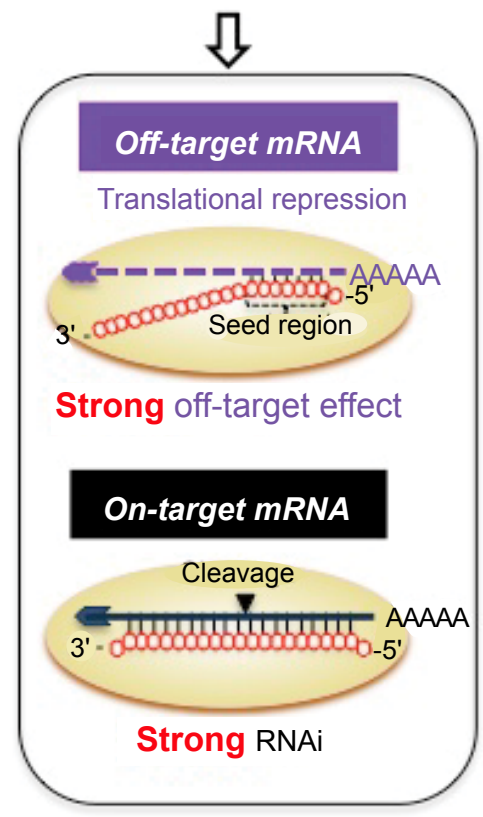

(b)
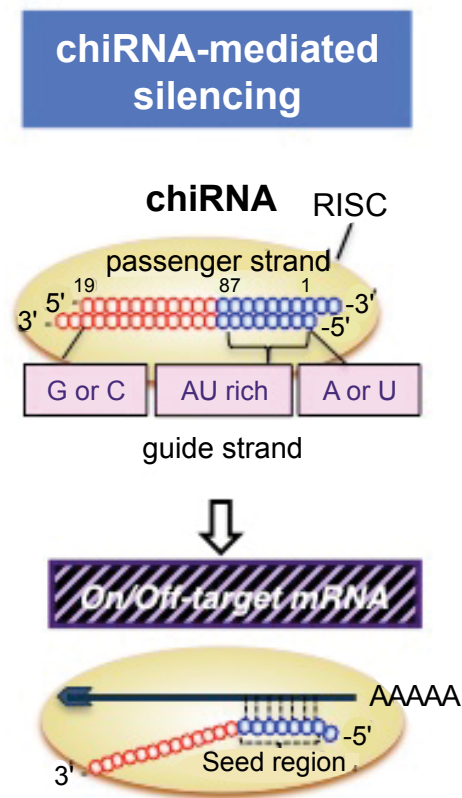

Weak base-pairing

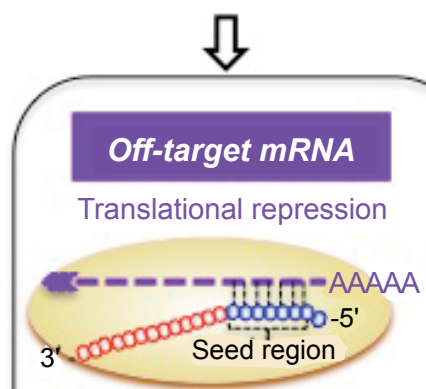

Weak off-target effect

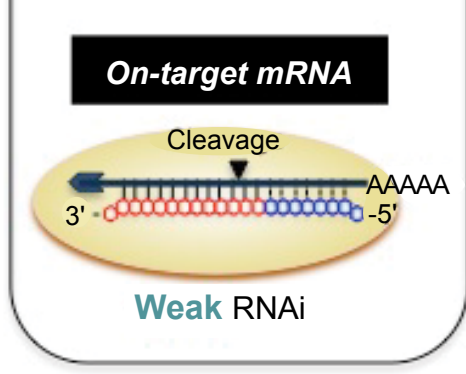

(c)
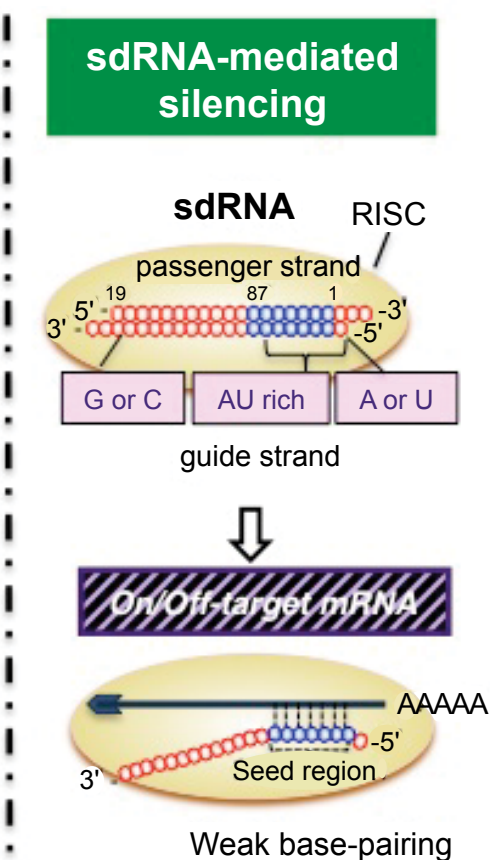

Weak base-pairing

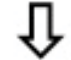

Off-target mRNA

Translational repression

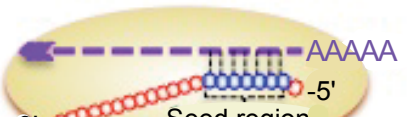

3. 000000000000000000000

Weak off-target effect

\section{On-target mRNA}

Cleavage

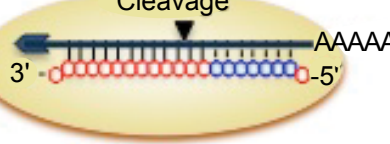

Strong RNAi

Figure 1: Comparison of the RNAi activities and off-target effects of (a) siRNA, (b) chiRNA and (c) sdRNA. The highly functional double-stranded small RNAs used in this study contained $A$ or $U$ residues at nucleotide position 1, four to seven A/Us at nucleotide positions 1-7, G/C at position 19, and a GC stretch of no more than nine nucleotides is not contained. The small double-stranded RNA unwound into a single-stranded RNA in the RISC. The guide strand retained in the RISC recognized on- and off-target mRNAs with complementary sequences to the seed region at positions 2-8 from the 5 ' end of the guide strand. The on-target mRNAs had perfectly complementary sequences. However, off-target effects were observed for mRNAs with seed-complementary sequences. The siRNA showed a strong off-target effect and strong RNAi activity. The chiRNA showed a weak off-target effect and weak RNAi activity. The sdRNA was ideal showing a weak offtarget effect and strong RNAi activity.

$24 \mathrm{~h}$ after transfection and their luc activity was measured using a Dual-Luciferase Reporter Assay System (Promega). The siRNAs used in this study were chemically synthesized (Sigma) (see S1). siGY-441, an siRNA for GFP knockdown, was used as a control. $\mathrm{IC}_{50}$ was determined from the results shown in figures 2 and 3 by using the following formula:

$$
I C_{50}=10^{\wedge}[\log (A / B) \times(50-C) /(D-C)+\log (B)]
$$

where in $A$ : of the two points on each Figure which bracket 50\% inhibition, the higher concentration of siRNA, $B$ : of the two points on each Figure which bracket $50 \%$ inhibition, the lower concentration of siRNA, $C$ : inhibitory activity (\%) at the concentration $B, D$ : inhibitory activity (\%) at the concentration $A$. 
(a)
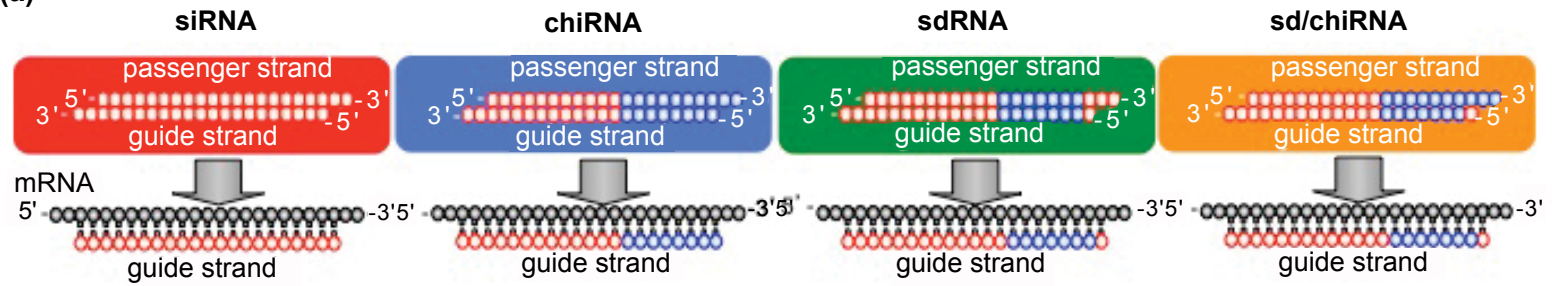

(b) CLTC2416

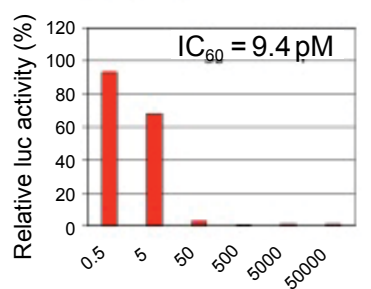

(c) FL2-153

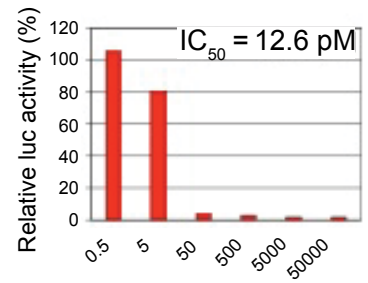

(d) FL3-36

siFL2-153 (pM)

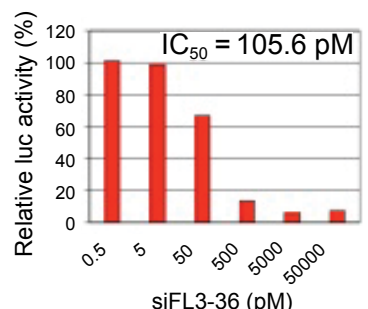

(e) VIM270

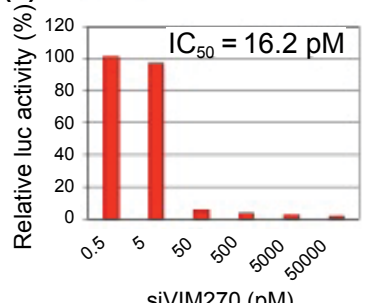

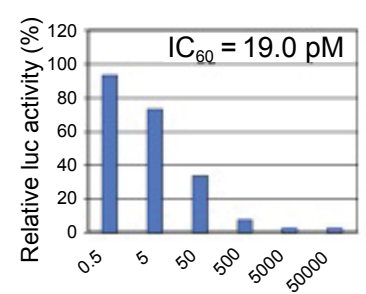

chiCLTC2416 (pM)

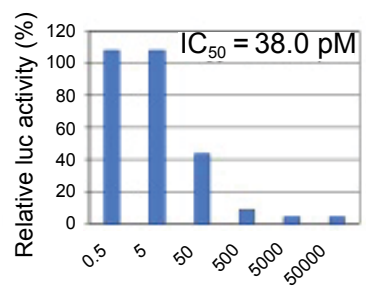

chiFL2-153 (pM)

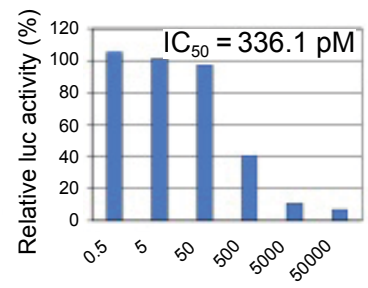

chiFL3-36 (pM)

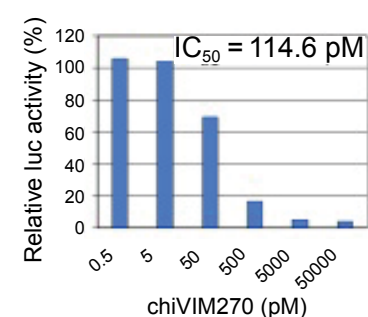

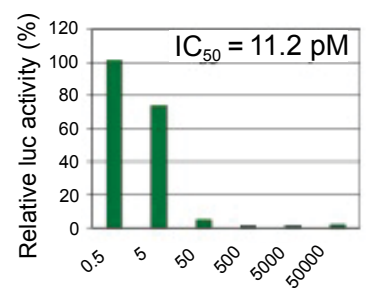

sdCLTC2416 (pM)

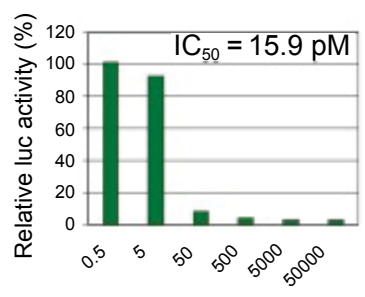

sdFL2-153 (pM)
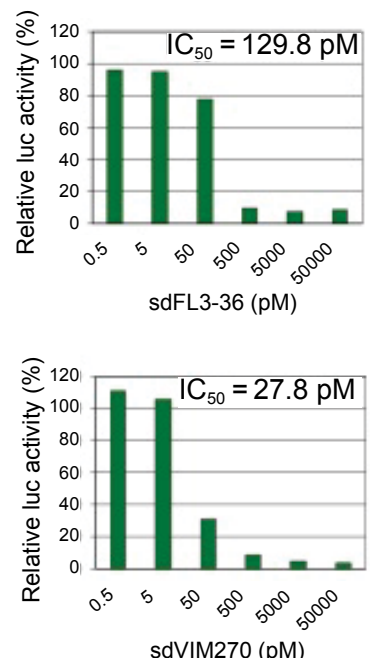

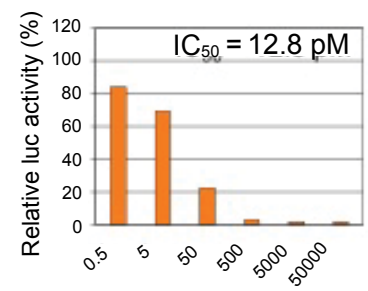

sd/chiCLTC2416 (pM)
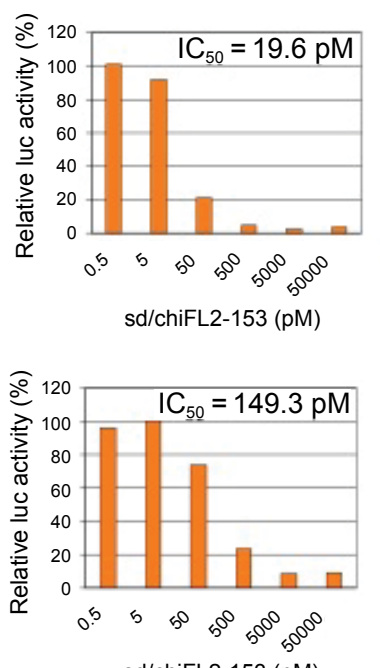

$\mathrm{sd} / \mathrm{chiFL} 2-153(\mathrm{pM})$

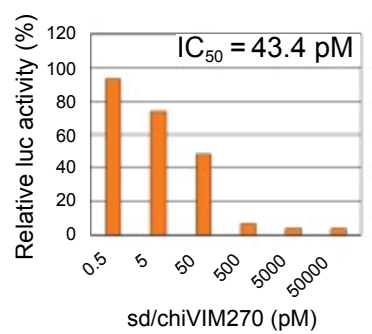

Figure 2: RNAi activity assay using reporter plasmids containing the CM target sequence. (a) Structures of the siRNAs, chiRNAs, sdRNAs, and sd/chiRNAs and their base-pairing patterns with completely matched targets; RNAi activities of the siRNAs, chiRNAs, sdRNAs, and sd/chiRNAs of (b) CLTC2416; (c) FL2-153; (d) FL3-36 and (e) VIM270.

\section{Plasmid construction}

All plasmids constructed were derivatives of psiCHECK-1 (Promega). Chemically synthesized oligodeoxynucleotides, including one copy of the 23-bp CM target sequences (S2) or three copies of the SM target sequences (S3) with cohesive XhoI/EcoRI ends, were inserted into the psiCHECK-1 (Promega) Xhol/EcoRI site, which is situated in the region encoding the $3^{\prime}$ UTR of Renilla luc mRNA; they are referred to as psiCHECK-CM and SM, respectively. The inserted CM or SM targets were expressed as part of the 3' UTR of Renilla luc mRNA in the transfected cells. The CM target completely matched the siRNA guide strand (Figure 2a), whereas the SM target consisted of two parts: the eight 3 ' terminal nucleotides of the target were complementary in sequence to the $5^{\prime}$ end (nucleotide position 1) and the seed (positions 2-8) of the corresponding siRNA guide strand, while the remaining thirteen nucleotides were totally non-homologous to the guide strand (Figure 3a).

\section{Results and Discussion}

\section{Designing sdRNA}

We developed an improved method using siRNAs with DNA in 
(a)

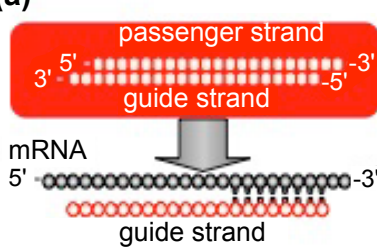

(b) CLTC2416

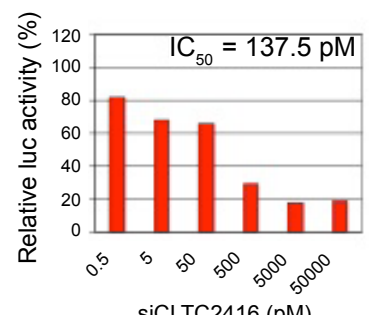

(c) FL2-153

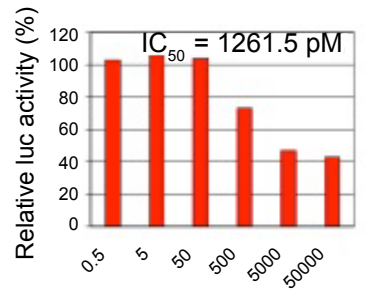

(d) FL3-36

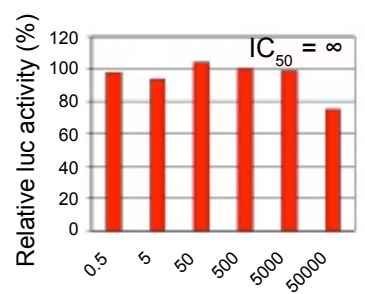

(e) $\operatorname{VIM} 270^{\mathrm{siFL} 3-36(\mathrm{pM})}$

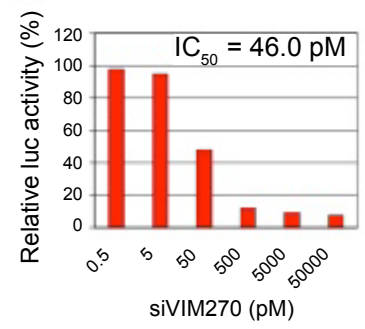

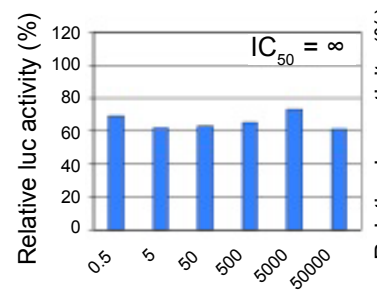

chiCLTC2416 (pM)

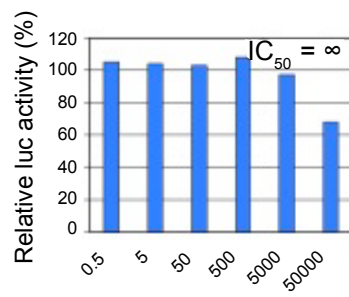

chiFL2-153 (pM)

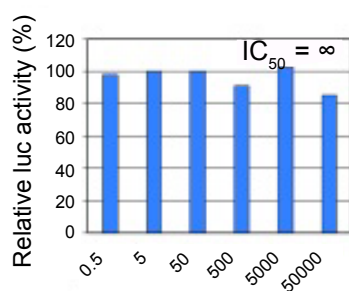

chiFL3-36 (pM)

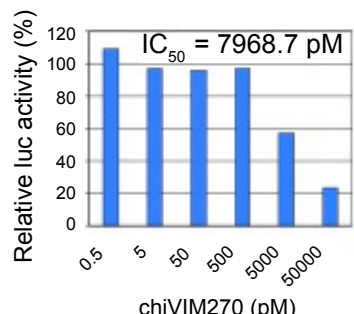

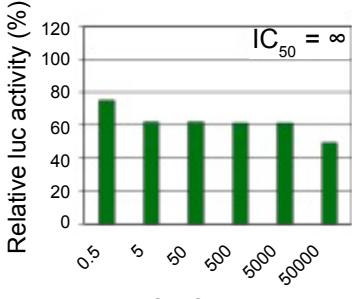

sdCLTC2416 (pM)

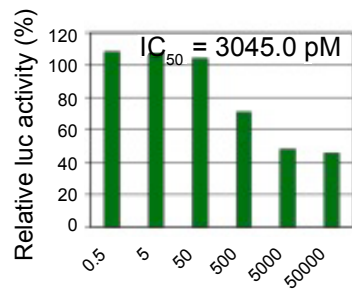

sdFL2-153 (pM)
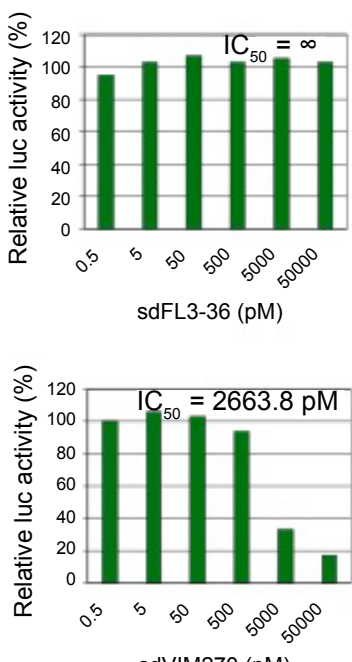

sdVIM270 (pM)
sd/chiRNA

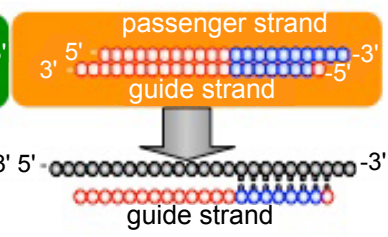

guide strand
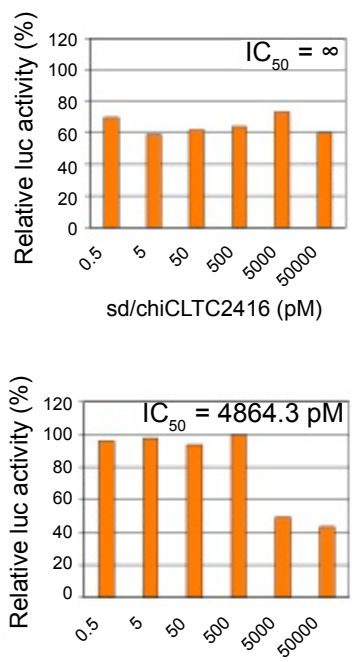

sd/chiFL2-153 (pM)
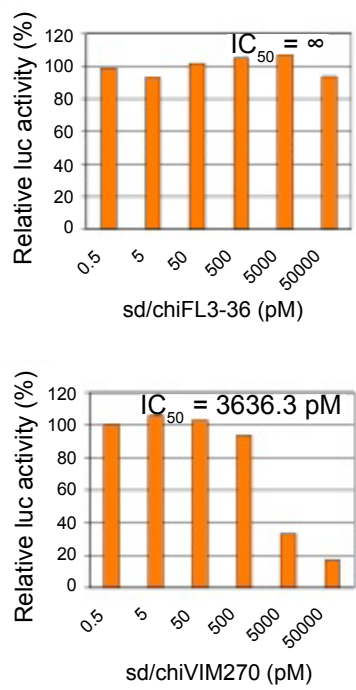

Figure 3: Off-target effect activity assay using a reporter plasmid containing SM target sequence. (a) Structures of the siRNAs, chiRNAs, sdRNAs, and sd/chiRNAs and their base-pairing patterns with seed-matched targets; Off-target effect activities of the siRNAs, chiRNAs, sdRNAs, and sd/chiRNAs of (b) CLTC2416; (c) FL2153; (d) FL3-36 and (e) VIM270.

the seed region (sdRNA), in which ribonucleotides were replaced with DNA at positions 2-8 from the 5 ' end of the siRNA guide strand but a ribonucleotide at the 5 terminal end was not replaced with DNA. The complementary ribonucleotides of the guide strand seed region in the passenger strand were also replaced with DNA (Figure 1c). The sdRNA was expected to have strong target-specific RNAi activity and weak offtarget effect activity, because it had a DNA seed sequence that could form a DNA-RNA seed-target duplex with low stability compared to that formed by an RNA-RNA duplex; the other positions (at 1 and 9-21) were ribonucleotides. In particular, A or $\mathrm{U}$ ribonucleotide residues at the $5^{\prime}$ end of the guide strand might be important because the $\mathrm{A} / \mathrm{U}$ nucleotide itself at the 5 terminal was shown to contribute strongly to RNAi activity. This observation might be explained by the fact that the nucleotide monophosphates AMP and UMP bind to Ago2 with up to 30-fold higher affinity than that observed with either CMP or GMP [24].

Comparison of the on-target RNAi activities of siRNAs, chiRNAs, sdRNAs, and sd/chiRNAs

To compare the RNAi activities of siRNAs, chiRNAs, and sdRNAs 

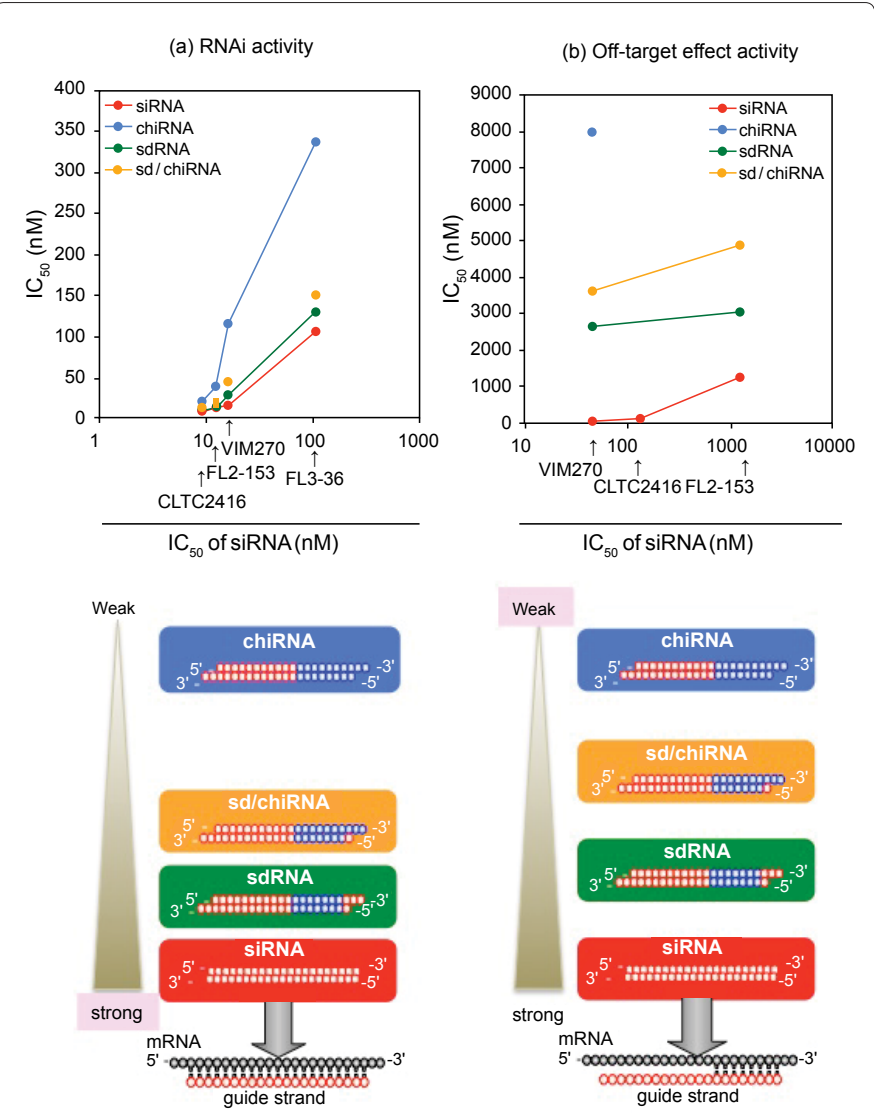

Figure 4: RNAi activities and off-target effect activities of the siRNAs, chiRNAs, sdRNAs, and sd/chiRNAs. Comparison of the (a) RNAi activities and (b) offtarget effect activities of the siRNAs, chiRNAs, sdRNAs, and sd/chiRNAs. Upper panels: The horizontal axis indicates the $I_{50}$ of each siRNA. Vertical axis indicates (a) IC of the RNAi activities and (b) off-target effect activities of siRNAs, chiRNAs, sdRNAs, or sd/chiRNAs with the same sequences as the siRNAs shown on the horizontal axis. Lower panel: schematic representation of the (a) RNAi activities and (b) off-target effect activities of the siRNAs, chiRNAs, sdRNAs, and sd/chiRNAs.

based on the same sequences (Figure 2a), four different sequences complementary to human clathrin heavy chain (CLTC2416), luc (FL2-153 and FL3-36), and human vimentin (VIM270) were used. As detailed in the Experimental Section, we constructed psiCHECK-CM reporters that contained Renilla luc with the complementary sequence of each siRNA introduced into its 3' UTR. The psiCHECK-CM plasmids were transfected into human HeLa cells together with pGL3-Control (encoding firefly $l u c$ ) along with small double-stranded RNA. One day after transfection, Renilla luc activity was measured with reference to the firefly $l u c$ activity level in the transfected cells, and the relative $l u c$ activities were calculated. Each of the highly functional siRNAs reduced the relative luc activity (RNAi activity) in a dose-dependent manner at siRNA concentrations of $0.5 \mathrm{pM}$ to $50 \mathrm{nM}$ (Figure $2 \mathrm{~b}-\mathrm{e}$ ). The calculated $\mathrm{IC}_{50}$ values of the nonmodified siRNAs varied from 9.4-105.6 pM depending on the sequence, indicating that siRNAs used in this study are very active as reported by others $[25,26]$. The $\mathrm{IC}_{50}$ values of the chiRNAs were from 19.0-336.1 pM, a decrease of $14-49 \%$ compared to the $\mathrm{IC}_{50}$ values of the siRNAs. The $\mathrm{IC}_{50}$ values of the sdRNAs were from 11.2-129.8 pM, about 58-83\% of those of the siRNAs. As shown in figure $4 \mathrm{a}$, siRNAs showed the strongest RNAi activities in all of four siRNAs used in this study, while chiRNAs showed the lowest activities. Because sdRNAs with a deoxyribonucleotide in the seed region showed equivalent RNAi activity with the siRNAs, the low RNAi activity of the
chiRNAs might not be due to the deoxyribonucleotide substitutions in the seed region. To investigate whether a deoxyribonucleotide at the $5^{\prime}$ terminal end of the guide strand and three deoxyribonucleotides at the $3^{\prime}$ end of the passenger strand were involved in the reduction in RNAi activity, we examined the effect of an sd/chiRNA containing an sdRNA guide strand and chiRNA passenger strand. These sd/chiRNAs also showed equivalent RNAi activities with the siRNAs; their $\mathrm{IC}_{50}$ values were $37-73 \%$ of the siRNA values (Figure $2 \mathrm{~b}-\mathrm{e}$ ), suggesting that the 5 end ribonucleotide in the guide strand played an important role in the RNAi activity. These results show good agreement with the previously reported result that AMP and UMP bind to Ago2 with up to 30-fold higher affinity than either CMP or GMP [24].

\section{Comparison of the off-target effect activities of the siRNAs, chiRNAs, sdRNAs, and sd/chiRNAs}

To compare the off-target effect activities of siRNAs, chiRNAs, and sdRNAs (Figure 3a), four different sequences, CLTC2416, FL2153, FL3-36, and VIM270, were used. Off-target effect activities were measured using the psiCHECK-SM reporter system. These highly functional siRNAs, siCLTC2416, siFL2-153, siFL-36, and siVIM270, showed off-target effects in a dose-dependent manner until the siRNA concentration reached $50 \mathrm{nM}$ (Figure $3 \mathrm{~b}-\mathrm{e}$ ). The calculated $\mathrm{IC}_{50}$ values of the off-target effects in the nonmodified siRNAs, siCLTC2416, siFL2153, and siVIM270, varied from 46.0-1261.5 pM, and that of siFL336 was not determined. However, the off-target effects of the chiRNAs were considerably reduced as shown in our previous report [16]; their off-target effects were reduced to undetectable levels, and the $\mathrm{IC}_{50}$ value of chiVIM270 was decreased to $0.6 \%$ (7968.7 pM) of the $\mathrm{IC}_{50}$ value of siVIM270. Previously, we showed that ability to induce an off-target effect is correlated to the thermodynamic stability of seed-target duplex of siRNA [12]. The melting temperature value of RNA-RNA duplex at seed positions $2-8\left(\mathrm{Tm}_{2.8}\right)$ of siCLTC2416 is $33.2^{\circ} \mathrm{C}$, which decreases to $12.7^{\circ} \mathrm{C}$ when DNA-RNA duplex is formed by chiCLTC2416, indicating the stability of seed-target duplex is strong for siCLTC2416 but weak for chiCLTC2416 consistent with the results shown in figure 3b. Similarly, $\mathrm{Tm}_{2-8}$ values of RNA-RNA duplexes formed between siFL2-153, siFL3-36, and siVIM270 and each target mRNAs were $21.0-26.2^{\circ} \mathrm{C}$, and the values of DNA-RNA duplexes formed between chiFL2-153, chiFL3-36, and chiVIM270 and each target mRNAs decreased to $3.1-18.9^{\circ} \mathrm{C}$ (Figure $3 \mathrm{c}-\mathrm{e}$ ) [12]. Since the $\mathrm{Tm}_{2-8}$ values in the duplexes formed between sdRNAs and target mRNAs are same as those of DNA-RNA duplexes formed between chiRNAs and target mRNAs, it was unexpectedly that the sdRNAs showed strong off-target effects compared to those of chiRNAs. The $\mathrm{IC}_{50}$ values of sdFL2-153 and sdVIM270 were decreased to $41 \%(3045.0 \mathrm{pM})$ and $1.7 \%$ of the $\mathrm{IC}_{50}$ value of each siRNA, respectively. As shown in figure $4 \mathrm{~b}$, the three siRNAs showed the strongest off-target effect activities, while the chiRNAs showed the weakest off-target effect activities; the sdRNAs showed intermediate levels of off-target effect activity. The decreases in off-target effect activities of the sdRNAs compared to those of the siRNAs might be caused by the DNA substitutions in the seed region, suggesting that decreased stabilities in the seed-target duplexes reduced the off-target effects. However, the off-target effects of the sdRNAs increased compared to those of the chiRNAs, suggesting that the 5' end ribonucleotide of the guide strand and three ribonucleotides at the 3 end of the passenger strand contributed to the increase in off-target effects. Furthermore, the sd/chiRNAs showed weak or similar levels of off-target activities compared to those found with sdRNAs (Figure $4 \mathrm{~b}$ ). This result suggests that the $5^{\prime}$ end RNA of the guide strand contributed strongly to the off-target effect, while the three ribonucleotides at the 3 end of the passenger strand contributed weakly to this effect. 
Citation: Ui-Tei K (2012) sdRNA: siRNA with a DNA Seed for an Efficient and Target-gene Specific RNA Interference. Gene Technol 1:102. doi:10.4172/2329-6682.1000102

Page 6 of 6

\section{Conclusions}

Based on our previous reports, we expected that an sdRNA that included a DNA seed would be an appropriate tool for inducing targetspecific RNAi with reduced off-target effects. Our results confirm that the sdRNA showed weak off-target effect activity compared to siRNAs and strong RNAi activity compared to chiRNAs. A 5' end ribonucleotide in the siRNA guide strand contributed strongly to the off-target effects, whereas the three ribonucleotides at the 3 end of the siRNA passenger strand contributed weakly to the off-target effects. Our results indicate that sdRNAs might be a powerful tool for developing ideal RNAi experiments and therapeutic applications.

\section{Supplementary Information Files}

S1. Supplementary Information 1. RNA sequences.

S2. Supplementary Information 2. Target sequences in psiCHECKCM.

S3. Supplementary Information 3. Target sequences in psiCHECKSM.

\section{Acknowledgements}

I am grateful to Dr Kaoru Saigo and Dr Yukikazu Natori for their valuable comments and discussions throughout this study, and Kiyoko Shinohara for preparing the figures. I acknowledge the funding sources: the Ministry of Education, Culture, Sports, Science and Technology of Japan (MEXT) [gran numbers 21115004, 21310123, 23651200], the Cell Innovation Project (MEXT) and Core Research Project for Private University, matching fund subsidy. The author declares no competing interests.

\section{References}

1. Elbashir SM, Harborth J, Lendeckel W, Yalcin A, Weber K, et al. (2001) Duplexes of 21-nucleotide RNAs mediate RNA interference in cultured mammalian cells. Nature 411: 494-498.

2. Hutvagner G, Simard MJ (2008) Argonaute proteins: key players in RNA silencing. Nat Rev Mol Cell Biol 9: 22-32.

3. Jinek M, Doudna JA (2009) A three-dimensional view of the molecular machinery of RNA interference. Nature 457: 405-412.

4. Khvorova A, Reynolds A, Jayasena SD (2003) Functional siRNAs and miRNAs exhibit strand bias. Cell 115: 209-216.

5. Schwarz DS, Hutvágner G, Haley B, Zamore PD (2002) Evidence that siRNAs function as guides, not primers, in the Drosophila and human RNAi pathways. Mol Cell 10: 537-548.

6. Ui-Tei K, Naito Y, Takahashi F, Haraguchi T, Ohki-Hamazaki H, et al. (2004) Guidelines for the selection of highly effective siRNA sequences for mammalian and chick RNA interference. Nucleic Acids Res 32: 936-948.

7. Reynolds A, Leake D, Boese Q, Scaringe S, Marshall WS, et al. (2004) Rational siRNA design for RNA interference. Nat Biotechnol 22: 326-330.

8. Amarzguioui M, Prydz H (2004) An algorithm for selection of functional siRNA sequences. Biochem Biophys Res Commun 316: 1050-1058.

9. Liu J, Carmell MA, Rivas FV, Marsden CG, Thomson JM, et al. (2004) Argonaute2 is the catalytic engine of mammalian RNAi. Science 305: 14371441.

10. Meister G, Landthaler M, Patkaniowska A, Dorsett Y, Teng G, et al. (2004) Human Argonaute2 mediates RNA cleavage targeted by miRNAs and siRNAs. Mol Cell 15: 185-197.

11. Song JJ, Smith SK, Hannon GJ, Joshua-Tor L (2004) Crystal structure of Argonaute and its implications for RISC slicer activity. Science 305: 1434-1437.

12. Ui-Tei K, Naito Y, Nishi K, Juni A, Saigo K (2008) Thermodynamic stability and Watson-Crick base pairing in the seed duplex are major determinants of the efficiency of the siRNA-based off-target effect. Nucleic Acids Res 36: 71007109.

13. Birmingham A, Anderson EM, Reynolds A, Ilsley-Tyree D, Leake D, et al. (2006) 3' UTR seed matches, but not overall identity, are associated with RNAi offtargets. Nat Methods 3: 199-204.
14. Lin X, Ruan X, Anderson MG, McDowell JA, Kroeger PE, et al. (2005) siRNAmediated off-target gene silencing triggered by a $7 \mathrm{nt}$ complementation. Nucleic Acids Res 33: 4527-4535.

15. Jackson AL, Burchard J, Schelter J, Chau BN, Cleary M, et al. (2006) Widespread siRNA "off-target" transcript silencing mediated by seed region sequence complementarity. RNA 12: 1179-1187.

16. Ui-Tei K, Naito Y, Zenno S, Nishi K, Yamato K, et al. (2008) Functional dissection of siRNA sequence by systematic DNA substitution: modified siRNA with a DNA seed arm is a powerful tool for mammalian gene silencing with significantly reduced off-target effect. Nucleic Acids Res 36: 2136-2151.

17. Jackson AL, Bartz SR, Schelter J, Kobayashi SV, Burchard J, et al. (2003) Expression profiling reveals off-target gene regulation by RNAi. Nat Biotechnol 21: $635-637$

18. Scacheri PC, Rozenblatt-Rosen O, Caplen NJ, Wolfsberg TG, Umayam L, et al. (2004) Short interfering RNAs can induce unexpected and divergent changes in the levels of untargeted proteins in mammalian cells. Proc Natl Acad Sci USA 101: 1892-1897.

19. Lewis BP, Burge CB, Bartel DP (2005) Conserved seed pairing, often flanked by adenosines, indicates that thousands of human genes are microRNA targets. Cell 120: 15-20.

20. Lim LP, Lau NC, Garrett-Engele P, Grimson A, Schelter JM, et al. (2005) Microarray analysis shows that some microRNAs downregulate large numbers of target mRNAs. Nature 433: 769-773.

21. Grimson A, Farh KK, Johnston WK, Garrett-Engele P, Lim LP, et al. (2007) MicroRNA targeting specificity in mammals: determinants beyond seed pairing Mol Cell 27: 91-105.

22. Ma JB, Yuan YR, Meister G, Pei Y, Tuschl T, et al. (2005) Structural basis for 5 '-end-specific recognition of guide RNA by the $A$. fulgidus piwi protein. Nature 434: 666-670.

23. Yuan YR, Pei Y, Ma JB, Kuryavyi V, Zhadina M, et al. (2005) Crystal structure of A. aeolicus argonaute, a site-specific DNA-guided endoribonuclease, provides insights into RISC-mediated mRNA cleavage. Mol Cell 19: 405-419.

24. Frank F, Sonenberg N, Nagar B (2010) Structural basis for 5'-nucleotide basespecific recognition of guide RNA by human AGO2. Nature 465: 818-822.

25. Detzer A, Overhoff M, Wünsche W, Rompf M, Turner JJ, et al. (2009) Increased RNAi is related to intracellular release of siRNA via a covalently attached signal peptide. RNA 15: 627.

26. Novobrantseva TI, Borodovsky A, Wong J, Klevanov B, Zafari M, et al. (2012) Systemic RNAi-mediated gene silencing in nonhuman primate and rodent myeloid cells. Mol Ther Nucleic Acids 1: e4. 